\title{
ON OCULAR NYSTAGMUS AND THE LOCALIZA- TION OF SENSORY DATA DURING \\ DIZZINESS.
}

\author{
BY PROFESSOR EDWIN B. HOLT, \\ Harvard University.
}

If a person sitting with his eyes closed and his head in an upright position is slowly set in rotation about a vertical axis lying within or near his head, he meets with a considerable variety of sensory experience. All these sensory data contribute more or less toward the perception of motion, but in different ways. And these data fall into three general groups which it takes no extremely subtle introspection to distinguish.

1. Firstly, one distinguishes a group of sensations which proceed from extra-peripheral stimuli: currents of air are felt by their impact or their temperature, on any uncovered surfaces of skin; any source of light which can be dimly perceived through the eyelids or eye-bandages, will be seen intermittently as with each rotation the face is brought opposite the light; any source of sound will be heard alternately loud and faint as with each rotation an ear is twice presented to it. The air currents would of themselves give no clue to the movement of the person's own body, and they are ordinarily felt as somewhat irrelevant data, which hardly even tend to fuse with the other sensations of motion. The visual and the (doubly rapid) auditory intermittences become, with increasing speed of rotation, shythms of which the spatial or temporal significations are subject to considerable individual differences. Thus for my own introspection the visual intermittence becomes a temporal rhythm, while the auditory sensations become a hoop of sound lying horizontally about the head as a center, and having two spots of maximum loudness opposite each other, and two of minimum loudness midway between them. I have sometimes, though seldom, had from the visual intermittence a comparable hoop of light. In any case the person is aware of a relative motion 
between his body and the hoop of sound, or light, but which of these is at rest and which in rotation is so far ambiguous and is determined by the factors given in group 3. Both the visual and the auditory phenomena are readily isolated in introspection, and both are felt to be distinctly ' secondary criteria' of motion.

The three sorts of sensation so far mentioned may all be eliminated by fairly simple precautions (though they are also readily ignored by the observer), but another kind of sensation is neither so readily elimated, nor ignored, nor distinguished from the sensations of group 2. This kind comprises the tactual sensations mainly of the hands, back, thighs and soles of the feet, which vary with the inertia of the body and with the centrifugal moment induced by the rotation. These sensations, while less clearly a secondary criterion of motion, while more intimately associated, that is, with the sensations of group 2 and even of group 3, can still after some practice be distinguished as tactual sensations of varying strength.

2. Secondly, there are the sensations from proprio-ceptive organs (Sherrington, '06, p. I30) in joints, muscles and other tissues, which are stimulated (similarly to the last-named class of group $\mathrm{r}$ ) by the inertia of the trunk, limbs, internal organs, and even perhaps of the blood, and by their centrifugal moment. Sensations supposed to be stimulated in this way in the cerebellar mass or its sensitive coatings were originally adduced by Purkinje (Aubert, '88, S. II9-120) to explain dizziness ; and in connection with movements of translation Delage ('86, p. 623) has referred to sensations seemingly "produced by a sort of internal tidal movement in which all the liquids and such solid organs as have any mobility, participate." While such factors are hypothetical, certain sensations from proprio-ceptive organs in joints, tendons and muscles undoubtedly play a part in the perception of the motion of one's own body (Schäfer, '87; Breuer, '9o, S. 204; Mach, '73, S. I27; Abels, '06, S. $3^{82}$ ). It is difficult even after practice to distinguish introspectively these sensations from those of group 3, except when, with a high speed of rotation, they become intense, whereupon they are readily distinguishable as secondary criteria, from the true 
sensations of movement of group 3. How much these proprioceptive stimulations when not intense contribute to the perception of movement is hard to determine. Some writers, as Abels ('o6 and 'o7), have wished to find in them the very basis of that perception, but this is an unwarrantable view for as Mach ('74, S. 130) has said: "One can scarcely explain feelings of motion in terms of skin or muscle sensations, in view of the feelings in the head, the enormous influence of the head position, and Flourens's experiment:" and indeed Mach might have added, in view of well-nigh every fact that experiment has yielded regarding the canals and sacs of the ear (cf. also Breuer, 'o7). Or, as (Crum) Brown has said ('95, p. I5), "A few experiments . . . will convince any one that we have here to do with a perfectly definite sense, and not with any vague sensations caused by the inertia of the soft parts of the body." Nevertheless the proprio-ceptive sensations are of interest here, and deserve more experimental notice than they have so far had.

3. Thirdly, there are the true sensations of motion which are in some way dependent on the semicircular canals, and probably the sacs, of the ear. These present a remarkable complication of phenomena, with which we shall have chiefly to deal in the present paper. And firstly introspectively. If a person sitting with his eyes closed and head upright is rotated about a vertical axis within or near his head, and if the speed of rotation increases continuously, the person feels his body to be rotating in the direction of the actual motion, and he also generally feels objects in the space around him (by as much as he is aware of them) to be moving more or less rapidly in the opposite direction. Two things, in short, the body and the objects around it, are felt to be in relative motion.

Problem I. - What organs yield the sensation of rotation?

I believe that it has not so far been noticed that unless the rotation is very rapid, the direction of the attention is able to determine which of these shall be felt to be the more involved in motion and which to be almost or quite at rest. If, namely, the person 'directs his attention' to his own person, this will seem to be in rapid motion while then the environment may 
seem to be quite at rest : but if the attention is directed to the environment sweeping by, if, that is, the sensations of groups I and 2 occupy the focus of attention, the objects about the person will seem to whirl rapidly to the rear while his own body will seem to be nearly or quite at rest. I find also in this case a faint suggestion in consciousness of a space far behind these dimly presented objects, which is, like my body, at rest. When the rotation is rapid, however, it is much more difficult and often impossible to achieve such a 'setting' of the attention. Also, as we shall later see, some individual differences are to be expected in this field. This influence of the direction of the attention on the perception of motion during rotation is doubtless analogous with the effect mentioned by Hering of the same factor, on the apparent position of objects.

Problem II. - What is it which is involved in what we introspectively call 'setting the attention,' which in the case of rotation can shift the appearance of motion from one object to another?

If the rotation is long protracted at an ever-increasing rate of speed, the person becomes sick. This phase is no part of dizziness proper, and does not here concern us. The remaining introspective phenomena which interest us occur when the speed of rotation is decreasing, and after the rotation has stopped. Now when the acceleration of motion changes from positive, or zero, to negative, the person feels without appreciable latency both himself and the objects around him to be rotating in the contrary direction. Here, too, the direction of the attention is of influence, but here the attention is to be directed against the illusory motion, stemming the tide as it were, and then the movement both of the body and of such sensations as one has of the outer objects (group I) is alike diminished or annulled.

Problem $I I I$. - How does setting the attention against the illusory post-rotary movement reduce the apparent motion of both the body and the enviroment?

If now the eyes are opened, the apparent rotation persists (although cf. Bárány, 'o6, S. 223) save that the visual field is far more prominent than before; and it is still whirling con- 
trariwise to the original rotation. As Mach ('73, S. 127) has described it: "As soon as the apparatus is slowed down one has the feeling of making a contrary rotation together with the box [in which one is enclosed]. If now the box is opened, entire visual space with its contents rotates. It is as if all visible space were turning within a second space which one believes to be motionless, although it is identified by no visible cue. One might almost believe that there exists behind visual space another space to which the visual is always referred."

Furthermore, during the decrease or immediately after the end of the rotation, objects presented to the tactual sense are felt to be in contrary rotation, similar to that of the visual field. This tactual dizziness is far less pronounced than the visual, but is sufficiently attested by Purkinje ('20), Mach ('oo, S. 100), Wundt ('02, Bd. 2, S. 586) and others. There is likewise an auditory dizziness quite analogous to the preceding, whereby sources of (continuous) sound appear, after the rotation, to rotate contrariwise. As Münsterberg and Pierce ('94, p. 475) have described it : "If after the rotation, but while the eyes were still closed, the sound was given continuously for a time, it seemed to make the illusory movement too: it remained, that is, in constant orientation with the body." These visual and auditory phenomena experienced after rotation are not, of course, to be confused with the 'hoops' and other phenomena of group I experienced during rotation.

The foregoing phenomena immediately suggest the following problems :

Problem IV. - Why does the body appear to reverse its motion and to rotate contrariwise when the acceleration becomes negative and after the actual rotation has ceased?

Problem V. - Why after the rotation has ceased does the visual field continue to rotate contrariwise?

Problem VI. - Why do tactual and auditory impressions likewise continue to rotate contrariwise?

While the behavoir of the visual field has since the early seventies been referred in a general way to the ocular nystagmus which is normally induced by rotation, the connection between the two is still susceptible of elucidation: and I am not aware 
that any specific explanation has been offered for the tactual and auditory dizziness. It was with these problems in mind that $I$ undertook in the fall of 1908 some experiments, on which the following discussion is partly based. It is also based in part on some experiments with dizziness previously described by me ('o6), and on the literature of the subject.

\section{What Organs Yield the Sensation of Rotation?}

On this question a very large number of investigators have come, although in a very general way, to some appearance of agreement. In general nearly all assent to the hydrokinetic theory of Mach, Breuer and (Crum) Brown, ${ }^{1}$ and I believe that in the main this theory is established beyond all question while some of its details will bear further examination. The experimental facts show that the semicircular canals of the ear are stimulated by circular and rotary motions, and that the utricle and saccule are (almost certainly) stimulated by motion of translation. (Some writers dispute the latter point, although, as it seems to me, on bardly sufficient grounds.) And from this the conclusion is commonly drawn, that these organs yield directly the sensations of rotation and of translation. The argument is - (I) sensations of motion depend on the position of the head; (2) the only receptor organs situated in the head, which are stimulated by motion, are the ampullæ and sacs of the ear; (3) therefore the sensations of motion are sensations from the ampullæ and sacs. Now clearly the two premises warrant the conclusion that - therefore the sensations of motion result from stimulation of the ampullæ and sacs. But these sensations need not result directly, as the first conclusion affirms. Nor have I so far discovered any experiments adduced explicitly to show that sensations of motion result directly rather than indirectly, from labyrinthine stimulation, although the former is commonly assumed. Bárány ('o6, S. 265, S. 275-6) touches on this point and declares: "It is an error to say that excitations thereof [i.e., of the canals and sacs] do not come to consciousness; strong excitations of them do come to consciousness, either as

'This theory has been admirably summarized by Nagel ('05, S. 790) and by Peters ('os). 
such or in combination with [unter Mithilfe d.] the accompanying eye-movements" (eye-movement sensations?). But the ground of this affirmation is neither here nor elsewhere made clear. The only other reference to just this point, which I have found, is in Ewald ('92, S. r33): "There are not merely special physiological movements, which take place during and after rotation, but also these are accompanied by special sensations. But the relation existing between the two has never been made clear. It has been taken for granted that the abnormal movement ensues on an abnormal sensation, and is in a way its visible expression. But $I$ do not believe that the relation is such a simple one." And in another place (S. $\mathrm{I}_{4} \mathrm{I}$ ) Ewald finds the movements following rotation to be ' reflex,' by which he clearly means that they are produced directly by the labyrinthine excitations zuithout these latter having come to consciousness. There is, then, ground for debate in this matter.

On the other hand it has been generally granted that the nystagmic movements of the eyes are closely connected with the visual dizziness. Thus at the very outset Purkinje attributed visual dizziness to 'unconscious' eye-movements carried over [übertragen] to outer objects (Aubert, '88, S. II7). Delage, too, while deeming the labyrinth an organ of sensation in the strict sense, ascribes features of visual dizziness to movements of the eyes ('86, p. 6ro-II). Mach ('oo, S. 98-100), Breuer ('98, S. 499) and Kreidl (ibidem) also attribute visual dizziness and illusions as to the vertical, to nystagmic and compensatory eye-movements; although these writers too believe in direct labyrinthine sensations. The accepted view should seem to be, then, although I do not know of an explicit statement to this effect, that movements of one's own body in rotation and translation are perceived by means of sensations coming directly from the ampullæ and sacs, while visual illusions of rotation and many of motion and position are due to reflex (and ' unconscious') eye-movements. Motion of translation of one's own body would be perceived by means of sensations from the sacs, of rotation by sensations from the semicircular canals.

Now there stands in somewhat surprising contrast to this view the experimental fact that both during and after rotation 
the sensation of rotation of one's own body is instantly inhibited if the ocular nystagmus is inhibited. This observation was first made by Bárány ('o6, S. 224): "The direction of one's line of regard is also of infuence on the illusory sensation of rotation. If $I$ have nystagmus horizontalis to the right $[i . e$., the rapid eye jerk toward the right and slow movement toward the left], which I can inhibit by looking toward the left, and if with my eyes closed I do look toward the left, the sensation of apparent rotation stops at once-just as the apparent motion of outer objects had [previously and for the same reason] stopped. If I look again to the right, the illusory motion commences again. One can observe several such disappearances and reappearances of the sensation of rotation. A considerable number of physicians was able to observe this phenomenon, which I am the first to describe, and I have had the same reports from enquiries among patients."

I have previously reported ('o6, p. 72) that the slow phase of the ocular nystagmus can " not voluntarily be inhibited; whereas the swift movement is so far voluntary that it can be inhibited at pleasure. It is possible, that is, to fix the eyes on that side of the field toward which the slow movements are directed, but not on any point at the other side of the field." And this inhibition of the nystagmus always inhibited visual dizziness; but $I$ had not at that time noticed that it also inhibits the apparent rotation of one's own body. Now conflicting statements are to be found on this point, and the most emphatic are those of Mach, who states ('74, S. 123) "that a person can have very marked subjective phenomena of rotation with demonstrably fast fixation and no eye-movements. If on the inside of the paper box described in the previous communication [the observer was inside the box, and both rotated] there is fastened a black cross on a white ground, so that when one fixates the crossing every deviation of the line of regard is betrayed by an after-image, then one observes no such afterimage when dizziness starts up. One can fixate and still feel dizzy. I have also convinced myself by direct observation of the eyes of a second observer, that the eyes can remain at rest when the experiment is carried out in the way I have described." 
And twenty-six years later Mach again wrote ('oo, S. IOI-2) to the same effect, although here it is visual dizziness rather than the sensation of bodily rotation which is not inhibited by voluntary fixation of the eyes. For Mach both kinds of dizziness undoubtedly persist.

It chanced that a few weeks before learning about the observations of Bárány, two other observers, Dr. Tait and Mr. Ricker, and myself noticed (accidentally, for we were then interested in visual dizziness) that voluntarily inhibiting the nystagmus does away with the sense of bodily rotation not merely after the rotation has stopped, but during the actual rolation itself. We were all three able repeatedly to undergo a lively passive rotation (axis of rotation vertical, head erect and over axis, eyes closed) of one to two minutes without at any time having the sensation of bodily rotation.

Such an inhibition of nystagmus throughout the experiment can be accomplished only in this way: Before the chair is set in motion (by a second person) the observer directs his fixation as far as possible in the direction contrary to the coming rotation, and holds his eyes in this position as long as the acceleration remains positive. With the eyes closed, as here, this requires some practice and we found that it could be facilitated by securing a fairly durable after-image on the retinæ immediately before the experiment. When the acceleration has nearly reached zero, $i . e$., when the speed has become nearly constant the observer relaxes his fixation and lets his eyes do as they will. They wander slowly toward the primary position of regard and remain there as long as the acceleration stays at zero. No motion of the body is felt if the voluntary control of the eyes is relinquished at the right moment. As soon, now, as the motion begins to be reduced (the acceleration is negative) the eyes wander involuntarily to the other side, $i . e_{\text {., with the actual }}$ rotation, and here they must voluntarily again be fixed until several seconds after the rotation has actually ceased. The experiment requires that the observer shall not actively assist to rotate himself.

If this is successfully accomplished all sensations belonging to group 3 are inhibited leaving, however, those of groups I 
and 2. The 'hoops' (group I) continue to rotate contrariwise as long as the actual movement lasts, but no longer (although they would continue contrariwise still longer if the nystagmus were not inhibited), and the centrifugal sensations are distinct in consciousness; and yet so insignificant are these secondary criteria of motion as compared with the primary sensations thereof, that the subject feels himself to be at rest in a somewhat remote though whirling entourage. This motion of surrounding objects is far from being adequately realized, as we ascertained occasionally by opening the eyes during rotation, whereupon the sudden realization of the rapid movement (backward and contrary to the actual movement) of visible objects came as a shock. But even this does not reinstate the sense of one's body being in motion provided that the nystagmus is still inhibited. It startles one sometimes into relaxing the hold on one's chair, so that we found it to be very disagreeable and somewhat risky to open the eyes while the nystagmus (and therewith the feeling of rotation) were being suppressed and the rate of rotation was rapid. The three observers above mentioned were well trained in the observation of dizziness; another subject, with less training and rather easily nauseated by dizziness, underwent the rotation without a sense of being himself in motion: and two women, quite untrained in the matter of dizziness, suppressed the post-rotary feelings of bodily rotation on the first trial, by inhibiting the post-rotary nystagmus; and on second trial succeeded in feeling no motion during as well as after the rotation. In all the experiments I observed nothing which would lead to any other conclusion than that voluntary inhibition of the ocular nystagmus directly inhibits the sensation of the rotation of one's own body.

These experiments go wholly to confirm the observation of Bárány which was given above. And we must now consider the precisely contradictory testimony of Mach. Since there is not the slightest ambiguity in the form of his statements, there remain three conceivable ways of reconciling them with the other observations above reported. It is possible that with Mach and his subjects the nystagmus was not really inhibited, for not all who try to inhibit it succeed. This is rendered 
plausible by the fact that Mach nowhere speaks of inhibiting the nystagmus by fixating toward the side contrary to motion, and in the absence of such a statement one must suppose that the inhibition of nystagmus was attempted by trying to hold the eyes voluntarily in the primary position, $i . e$., straight forward (cf. the above quotation from Mach). Now I am personally quite unable to inhibit the nystagmus, either during or after rotation, in this way, nor have I seen another person who was able to do this; and it is clear from Bárány's observations ('o6, S. 215-17) that such an attempted fixation straight ahead might actually augment rather than decrease the nystagmus. Nor can I attach much importance to the after-image test above quoted, with the black cross on a white ground, since there is no vision during the quick phase of the nystagmus, as we shall see later, and since the slow phase is too slow to leave a perceptible after-image streak unless the stimulus (here the ' white ground ') is very intense.

Yet aside from this Mach says that he examined the eyes of another observer during the voluntary inhibition. And while this is very difficult, since Mach himself must also rotate, while also nystagmic movements sufficient to produce dizziness can be so minute as to need a reading telescope for their discovery (Bárány, 'o6, S. 214), I cannot think it probable that Mach would have convinced himself, as he says, that this observer had inhibited the nystagmus if such had not really been the case.

A second possibility would be that Mach and his subjects mistook centrifugal sensations (group 2) for the true movement sensations of group 3. (Sensations of group I give no feeling of bodily motion so long as the nystagmus is inhibited.) This would be very probable with observers of little experience, but it can hardly have happened with $\mathrm{Mach}$; and furthermore he says that visual dizziness also continues after the inhibition of nystagmus, and centrifugal sensations could scarcely have been mistaken for visual dizziness.

The third alternative remains, that we have here a true case of individual difference. And one must be the more willing to admit this here since it is not more remarkable than other mani- 
fest discrepancies among the observations of careful experimenters in this same field. Thus Bárány, for instance, gets post-rotary dizziness of his body so long as his eyes are closed, but this is supplanted by visual dizziness when he opens his eyes: whereas Mach, and most other observers, feel with the eyes open both kinds of post-rotary dizziness at once (Bárány, 'o6, S. 223). Or again, in post-rotary visual dizziness Bárány sees the visual field oscillate from side to side in both directions ('o6, S. 22I); whereas Mach, Breuer, Delage and most other observers see it whirl contrariwise to the preceding actual rotation (Nagel, 'o5; Peters, '05); and Helmholtz ('67, S. 603; '96, S. 747) saw it whirl sometimes with and sometimes contrary to, the direction of the preceding rotation. Still more extraordinary are the different observations as to the localization of a visual after-image with the eyes closed, during voluntary and involuntary eye-movements. Indeed there are few branches of psychology where entirely credible observers more widely disagree regarding simple matters of fact. And I should designate this branch as the one comprising the following four things and their interrelations - motion, muscular contraction, the voluntary innervation to contraction, and the perception of movement.

Granted, then, the fact of unusual individual or typical differences, it remains to study the several types in and for themselves, in the anticipation that in the end some explanation will be found which will reconcile all discrepancies. I have not so far seen a subject who, like Mach, experiences bodily and visual dizziness after he has inhibited his ocular nystagmus, but I shall look for such persons, and meanwhile return to the discussion of such cases as Bárány and $I$ have met. For some things are inevitably true of these subjects, whatsoever else may be true of the members of other types. Now we have seen that for the subjects who are at present in question, voluntary inhibition of the nystagmus inhibits the sensation of bodily rotation. Bárány ('o6, S. 275-6) has sought to interpret this fact: "We have further seen that inhibition of the nystagmus elıminates the sensation of rotation. Since the voluntary direction of the regard can scarcely effect an inhibition of impulses coming over 
the vestibular nerve, we seem bound to conclude that the nystagmus as such, the involuntary and unconscious movement of the eyes, is of influence on the production of the rotary sensation; perhaps, indeed, that it is the nystagmus center in which the abovementioned integration [Verarbeitung] of the vestibular impulses and the excitations occasioned by changes of the head position, takes place. I perhaps went too far in an eàrlier paper, where I said that inhibition of the rotary sensation through inhibition of the nystagmus, proves that the nystagmus and not the vestibular impulses cause the rotary sensation. It is sufficient to assume that for the production of rotary sensation such impulses are necessary as, ozving to the nystagmus, are dclivered to the center involved, and that these form so lurge a component in the integration of the subcortical impulses [?] that the inhibition of then suffices to prevent the sensation of rotation from being produced. Nothing but a case of total, bilateral, oculomotor paralysis of central origin could really decide the point: in such a case there ought to be no sensation of rotation." I quote this passage in full (with italics as in the original) because it bears so explicity on our theme. I understand Bárány's conception to be that afferent vestibular impulses and afferent impulses from eye-movements are combined in a subcortical center, from which they emerge in consciousness as the sensation of rotation : and that the latter components (which would ordinarily be called 'sensations of eye-movement') at least are indispensable to the production of rotary sensations. Presumably he would hold the vestibular impulses to be indispensable as well.

But there are alternative possibilities. Is it true, as he declares, that "the voluntary direction of the regard can scarcely effect an inhibition of impulses coming over the vestibular nerve"? The voluntary direction of the regard certainly inhibits whatever impulses those are which produce the rapid phase of the nystagmic movement, and I see nothing to warrant a statement on one side or the other as to the relation between the vestibular and the voluntary impulses. It might be that the vestibular sensations are the sensations of rotation, but that these are inhibited when the rapid eye jerks are inhibited. And yet on the one hand professed ignorance is better than so far- 
fetched and mysterious an assumption as this latter; while on the other hand, regarding the former assumption, we must remember the many cases in which one's body is felt to move, with sensations distinctly like those of group 3 , in which neither the semicircular canals nor the sacs can be supposed to be stimulated. A person who stands on a bridge and watches the water flow beneath, from time to time feels himself moving contrary to the flow of the water (Mach, 'oo, S. 104). Helmholtz ('96, S. 763-4) mentions that when the dome of an astronomical observatory is turned about, a person standing beneath it is apt to feel the floor and himself rotating contrariwise. And there are many other such illusions of bodily translation or rotation, not distinguishable from the sensations of group 3 , in all of which the stimulus is purely visual and there are no afferent vestibular impulses. These considerations, I believe, quite shut out the vestibular impulses from being essential to the sensation of motion of one's own body.

We have next to examine Bárány's second and indispensable component - the afferent impulses occasioned by the nystagmic movements. Before we can suppose such impulses to be essential, or even in any wise contributory to the perception of motion, we must answer satisfactorily the arguments so cogently, and one might almost say savagely, stated by Hering ('6r, S. $3^{0-32)}$ : They are, he says, "proof enough that only the displacement of retinal images, and not sensations of tensions in the muscles, acquaint me with changes in the position of my eyes." In short, Hering allows no share at all to eye-muscle sensations in the perception of eye-movement; and he is disinclined to allow even their existence. Is it, then, conceivable that they afford sensations of movements of the whole body? We must also bear in mind that the famous discussion between Plateau, Oppel, Helmholtz, Dvoŕák and others did not confirm the belief in the existence of eye-muscle sensations. We have also had recently, from Dodge ('O4 and 'o7), Judd ('05) and other investigators in the Yale Laboratory, fresh evidence that sensations of eye-movement play little or no part in the perception of space. Personally I am unable at present to dispense with ' eye-movement sensations' as a part of my psychological 
furniture, and yet in the present case I must admit that several facts seem to exclude them from assuming any importance. It is, apparently, the efferent motor impulses to the eyes rather than afferent impulses yielded by eye-movements that have taken place, that most closely parallel, or as some persons might say, are, the sensations of bodily movement.

These facts are, firstly, that of the two phases exhibited by the nystagmus, the rapid and the slow, one phase but not the other seems to cause sensations of bodily movement. This is the rapid phase. Under positive acceleration the rapid eyejerk is with the rotation, and in this same direction the body is felt to turn; and as soon as the acceleration becomes negative the rapid jerk takes place contrary to rotation, and the body is felt to reverse its motion although it is actually rotating in the same direction. At high but uniform speed, of course, there is no nystagmus and likewise no sensation of one's body being in motion. As Bárány has said ('06, S. 225), "the apparent rotation of one's own body is always in the same direction as the rapid nystagmic movement." Now on the sensory side there is nothing, so far as we know at present, to distinguish these movements so sharply from each other; for of course the circumstance that one is fast and the other slow would not account for one of them being 'sensed' and the other not. But on the efferent side there is a prime distinction - the motor impulses to the rapid novement can be voluntarily inhibited, while those to the slow cannot be checked (Holt, 'o6, p. 72). The voluntary attempt to inhibit them, which has to be made by trying to fix the regard in a direction wholly or partly opposed to the direction of the slow movement, results only in increasing the nystagmus (Bárány, 'o6, S. 215-17). (This is why Mach is trying to fixate a point straight in front, is unusually fortunate if he succeeded in inhibiting the nystagmus.) Now certainly the rapid movement cannot be called a voluntary movement, since the whole nystagmus arises involuntarily; but since the rapid phase is amenable to voluntary inhibition, it may properly be called semi-voluntary. Now this circumstance that the rapid phase which alone counts toward the sensation of bodily movement is more nearly related to voluntary effort than the other 
phase, is directly in line with those facts already referred to, which Hering so emphasized ('6r, S. 30). "A position of the eyes which I have not voluntarily induced, which therefore I did not already know pretty exactly before it took place, and even more a movement which takes place without my special intention - I am totally unable to estimate. ... [S. 3I] If I have voluntarily brought about a position of the eyes, then of course I know beforehand the direction and approximate extent of movement [involved], since otherwise I should not have been able to will just this movement; and both the direction and approximately the amount of force which is necessary for a given movement, are decided [bestimmt] by the will." This is coming, perhaps, very close to 'innervation feelings.' The empirical data to which Hering refers, may be summarized as the general lack of relation between the position of the eyes and the subjective localization of optical impressions. The eye-movement sensations, supposing them to exist, in many cases so inadequately register the eye-movements that a false localization is assigned to the objective sources of visual data. An example will sufficiently illustrate this general phenomenon.

In a previous paper ('o6, p. 72) I observed, " it is well known that after-images move with every involuntary eyemovement." Here I relied chiefly on the introspection during dizziness (with eyelids closed) of myself and four other subjects (ibid., p. 70-1), on some observations of my own on the vision of after-images during ' pursuit movements' (Dodge, 'o3), and I think on some printed statements which, however, I can no longer identify. My own observations were, and on retrial still are, unequivocal. But I have since discovered that other observers equally 'well know' that for them after-images do not move with involuntary eye-movements : on this point both Hering ('6I, S. 30-3I) and Bárány ('o6, S. 22I-2) are perfectly explicit, and several other authors imply the same view. On the other hand Hering's account distinctly implies that afterimages do move with voluntary eye-movements even when the eyelids are closed. Bárány ('o6, S. 222) gives this as Hering's view and confirms it himself (S. 221 ), Exner ('90, S. 50) and A. Nagel ('7I, S. 256) affirm the very same. Whereas 
Purkinje (Aubert, '88, S. I I8) recounts and apparently confirms an experiment of Charles Bell's in which " a blindingly bright image left on the eye after gazing on a shining object, always appears at rest during eye-movements executed in total darkness, and starts into motion only when the eyes are open and the [after-] image can be compared with external objects which are at rest." Now the observations in question are singularly easy to make, even for a novice, and I believe that such extraordinary discrepancies again rest on true differences between observers. (A colleague of long experience in the study of vision tells me that he gets both of the last-mentioned phenomena, but more commonly the latter of these.) Now these discrepancies, if we accept them, prove that there is no direct relation between eye-movements and the localization of afterimages, and such a thing is scarcely possible if visual data in general are localized by means of eye-movement sensations. But the main differentia, of which we know, between the above cases lies in the manner of innervation - whether this is voluntary, semi-voluntary, or involuntary : the first alone affecting the localization of after-images for Hering and Bárány. And with this view Mach ('oo, S. 93-105) also concurs. It is true that this does not remove all disagreement, for I find on myself and four subjects, that localization of after-images shifts with both phases (semi-voluntary and voluntary) of labyrinthine nystagmus, while Hering and Bárány deny this; and Purkinje and Bell find that even voluntary innervation does not affect the localization of after-images. Yet it is, to my mind, simpler to suppose that these divergencies rest on peculiarities of innervation than of eye-movement sensation. And in this Bárány, even more emphatically Hering, and also Mach would agree.

One can of course assume the inhibition, under given circumstances, of the supposed eye-movement sensations and hence their failure to govern localization; but there is nothing to inhibit them in our examples above save the several modes of innervation: so that again the explanation of the phenomenon would lie in peculiarities of innervation. Furthermore the observation of Mach, already described, that the feeling of both bodily and visual dizziness (rotary localization) can persist when 
there are no nystagmic movements could not be explained in terms of sensation of these non-occurring movements. And if recourse is then had to reproduced sensations of eye-movement, it again appears that only the labyrinthine or voluntary innervations could be effecting such reproduction. The fact that Lotze, Münsterberg and others have declared 'innervation feelings' to be reproduced sensations of movement does not affect the present case for the issue here is between afferent and efferent process, - whether incoming or outgoing impulses are more nearly parallel to feelings of motion. To resort, then, to reproduced kinæsthetic sensations is to yield the point at once and to grant that it is outgoing impulses and not impulses coming in from the eye-movements that govern the consciousness of movement. And while in Mach's experiment his feeling of bodily and visual dizziness cannot have come from sensations of eye-movement, since he says that the eyes were not moving, it may well have come from innervations to eye-movement, innervations which became inhibited at some level lower than their point of origin. It is worth noting that this view, and so far as I know no other view, accounts for the familiar pathological cases in which the innervation to contract a muscle which is paralyzed, produces the feeling of the intended movement although the muscle is not actually contracted by the innervation.

If now visual localization is not explicable in terms of eyemovement sensations, the localization of one's body is of course even less so. And thus this latter depends neither on afferent impulses from the eye-balls nor, as we saw before, on afferent impulses from the labyrinth; and yet the voluntary inhibition of eye nystagmus inhibits the feeling of movement (changing localization) of one's body. Only one conclusion remains the voluntary innervation to inhibit the nystagmus, which is really directed as we have seen to inhibiting the rapid phase of the nystagmus, suppresses the feeling of bodily motion by inhibiting the (semi-voluntary) innervation of that rapid phase. And it will be recalled that the feeling of motion of one's own body is always in the same direction as the rapid phase. Hence it is the semi-voluntary innervation to the rapid nystagmic phase 
which is the process most closely parallel to the feeling of bodily rotation. Our first problem was: "What organs yield the sensation of rotation?" And the answer would be that this is not a sensation in the ordinary meaning of that word, but that the process most nearly parallel to the fecling of rotation is one kind of innervation process. And I believe that this proposition applies to more than rotation, applies at least to all feelings of motion of one's body that are supposed to be given by the semicircular canals.

This result is nearly in line with a view long supported by Mach ('oo, S. 95), who says: "The will to execute movements of regard [Blickbewegungen] or the innervation (?) to these, is the very sensation of space itself." The question-mark after the word 'innervation' is presumably out of deference to those who oppose ' innervation feelings.' I should not care to say innervations are sensations of space nor, for reasons too general to be discussed here, that innervations are the feelings of bodily movement. The phrasing as italicized above is, I think, a somewhat securer statement. But it is clear that our argument, based in several places on observations at variance with those of Mach, comes out to a position not so far removed from his.

It is true that $I$ have not explained all the conflicting observations given above, nor have I, by-the-way, begun to exhaust the anomalies that stand on record in this field. But $I$ believe that the cases which we have considered, if they are facts and not errors of observation on the part of one person or another, necessitate the conclusion to which we have arrived. And whatsoever conclusion other facts lead to, it will not be contradictory to this of ours. We have found empirical evidence of three grades of innervation - voluntary, semi-voluntary, and involuntary - and that these exert a different influence over the inhibition of the feeling of movement. It is therefore probable that different grades of innervation are of different value in producing the feeling of movement. The three grades of innervation doubtless emerge at higher and lower neural levels; but the neural levels are many and hence the grades of innervation may be many. On this, I think, we may base a reasonable 
hope of explaining the so-far complicated and seemingly conflicting mass of observations.

The further problems raised in the first part of this paper must be discussed in a sequel, and it here remains to say only this: The conclusion that innervations of one kind or another are the process most nearly parallel to feelings of bodily motion, does not, of course, imply that such efferent impulses are created from nothing, as say on the 'psychic plane.' The nervous energy that constitutes these innervations is released by impulses coming more or less immediately from the periphery. For the physiological unit of the nervous system is the reflex arc. And the issue raised by our empirical data between afferent (from the eye-muscles or orbit) and efferent (to the same) is, I apprehend, more precisely stated as follows. Is the nervous process which runs parallel to the consciousness of the rotation or translation of one's body, one in which the afferent or sensory impulses come wholly or mainly from eye-muscles (or orbit), and diverge in the central nervous system, passing out as diffuse innervations to various and so far unidentified members; or is the process one in which the afferent impulses come from various and so far unidentified members, and converge in the central nervous system, passing out as a unified and definite innervation to eye-movement? The latter alternative is the conclusion that we have reached.

ABRLS, H.

BIBLIOGRAPHY.

'o6. Ueber Nachempfindungen im Gebiete des kinästhetischen und statischen Sinnes. Zeitschr.f. Psych., 43, 1906, S. 268-289; S. 374-422.

'07. Ist der 'Nachschwindel' im Endorgan oder nervös bedingt? Ibid., AUBERT, $\mathrm{H}$. 45,1907, S. 85-91.

'88. Physiologische Studien über die Orientierung, Tübingen, I888. BáRÁNY, R.

'o6. Untersachungen über den vom Vestibularapparat des Ohres reflektorisch ausgelosten rhythmischen Nystagmus und seine BegleiterscheiBRROER, J. nungen, Monatsschr.f. Ohrenheilk., 40, 1906, S. 193-297.

'o7. Bemerkungen zu Dr. Hans Abels Abhendlang, etc. Zeitschr. $f$. Psych., 45, 1907, S. 78-84.

'go. Ueber die Function der Otolithen-Apparate. Pfiuger's Archiv f. d. ges. Physiol., 48, 1890, S. 195-306. 
Brown, A. Crum.

'95. The Relation between the Movements of the Eyes and the Movements of the Head. Robert Boyle Lecture, London, 1895 .

DELAGES, Y.

'86. Etudes expérimentales sur les illusions statiques et dynamiques de direction, etc. Archives de Zool. exp. et générale, 2 ième Série, 4, 1886, pp. 535-624.

DODGE, $\mathbf{R}$.

'03. Five Types of Eye Movement, etc. Amer.Jour. of Physiol., 8, I903, pp. 307-29.

'04. Participation of the Eye Movements in the Visual Perception of Motion. Psych. Rev., 2, 1904, p. r.

'07. An Experimental Study of Visual Fixation. Studies fr. the Pyych. Lab. of Wesleyan Univ., I, 1907, pp. I-95.

EwaId, J. R.

'92. Physiologische Untersuchungen uber das Endorgan des Nervus Octavus. Wresbaden, 1892 .

EXNER, S.

'go. Das Verschwinden der Nachbilder bei Augenbewegungen. Zeitschr. f. Psych., I, I890, S. 47-5I.

HFI,MHOL,TZ, H.

'67. Handbuch der Physiologischen Optik. Leipzig, 1867.

' 96. Handbuch der Physiologischen Optik. Hamburg und Leipzig, 1896. HRRING, E.

'6r.' Beiträge zur Physiologie. Leipzig, 1861.

HoLr, E. B.

'03. Eye-Movement and Central Anæsthesia. Harvard Psych. Studies, I, 1903, pp. 3-45.

'o6. Eye-Movements during Dizziness. Ibid., 2, 1906, pp. 57-66.

'o6. Vision during Dizziness. Ibid., pp. 67-73.

JUDD, C. $\mathbf{H}$.

'05. Movement and Consciousness. Yale Psych. Studies, New Series, I, 1905, pp. 199-226.

$\mathrm{MACH}, \mathrm{E}$.

'73. Physikalische Versuche über den Gleicbgewichtssinn des Menschen. Sitzungsber d. kais. Akad. d. Wiss. Wien, math.-natumw. Cl., 68, 3te Abth., 1873, S. 124-140

'74. Versuche uber den Gleichgewichtssinn. Ibid., 69, 2te Abth., 1874, S. $12 \mathrm{~J}-\mathrm{I} 35$

'oo. Analyse der Empfindungen. 2te Aufl., Jena, 1900.

MÚNSTERBerg, H., and PIERCE, A. H.

'94. The Localization of Sound. Psych. Rev., I, I894, pp. 46I-476. NAGEL, W.

'05. Die Lage-, Bewegungs- und Widerstandsempfindungen. Handb. d. Physiol. d. Menschen. Braunschweig, 2, 1905, S. 734-806.

PETERS, $\mathrm{W}$.

'05. Die Bewegungs- und Lageempfindungen. Archtv f.d.ges. Psych., 5 , 1905, S. $42-76$.

PIerce, A. H.

See Munsterberg, $\mathbf{H}$. 
PURknjJe.

'25. Viertes Bulletin d. naturw. Sektion d. schles. Gesellsch. f. väterl. Kultur, 1825.

'26. Zehntes ditto, 1826 .

'26. Zweites Bull, d. naturw. a. botan. Sektion d. schles. Gesellsch. f. vater1. Kultur, 1826. (The above three Bulletins are reprinted by Aubert, '88.)

SCHAFER, K.

'87. Ueber die Wahrnehmung eigener passiver Bewegungen durch den Muskelsinn. Pfiuger's Archivf. d.ges. Physiol., 4I, 1887, S. 566.

SHERRINGTON, C. S.

'o6. The Integrative Action of the Nervous System. New York, 1906. WONDT, W.

'02. Grundzüge der physiologischen Psychologie. 5te Auf., Leipzig 1902-3. 